\title{
Factores relacionados con el incumplimiento a citas concertadas de un grupo de pacientes hipertensos
}

\author{
R. Orueta, R. M. Gómez-Calcerrada*, S. Redondo**, M. Soto**, \\ G. AlEJANDRE**, J. LÓPEZ** \\ Médico de Familia. ${ }^{*}$ D.U.E. ${ }^{*}$ Residente de $3^{e r}$ año de Medicina de Familia. \\ Centro de Salud "Sillería". Toledo
}

\section{RESUMEN}

Fundamentos: el incumplimiento es una causa frecuente de fracaso de las actuaciones médicas, siendo la ausencia a los controles periódicos una forma importante del mismo

Objetivo: conocer el grado de incumplimiento a citas concertadas de los pacientes hipertensos y los factores implicados

Diseño: observacional analítico.

Material y métodos: se incluyeron los pacientes hipertensos controlados en consulta concertada, siendo seguidos cada uno de ellos durante 1 año. Se consideró incumplidor a aquel que faltó a más del 20\% de las citas acordadas. En caso de incum plimiento a una cita se le telefoneó para concertar nueva cita.

Resultados: fueron incluidos 129 pacientes (me dia de 7,3 controles en el año). Existió incumpli miento al 11,2\% de las consultas y fueron incum plidores el 24,8\% de los pacientes, si bien el 51,2\% faltó a alguna de las citas.

Fueron más incumplidores los varones (36,2 vs $18,3 \%)$ y los menores de 50 años $(66,7 \%$ de incum plidores). No se observaron diferencias en relación con nivel cultural, estado civil ni situación laboral, pero sí en relación con la convivencia $(45,5 \%$ los que vivian solos vs 20,2 y 22,2\% sívivian en fami lia o con otras personas). Los pacientes con in cumplimiento previo también fueron más incumpli dores (34,5 vs $16,9 \%)$.

No se evidenciaron diferencias en el cumpli miento en función de la consulta, hora de citación, intervalo entre las citas o distancia desde la última consulta a demanda.

Conclusiones: no existe un perfil claro del pa ciente hipertenso incumplidor a controles, pero
Related factors with non-compliance to arrange appointments of a group of hypertensive patients

\section{ABSTRACT}

Fundaments: non-compliance is a frecuent cause of medical actions'failure, being the absence to periodical controls a very important form of the same.

Objective: to know the non-compliance degree to arrange appointments of patients with arterial hypertension and the factors involve in it.

Design: observational, analitical.

Materials and methods: it was included all pa tients with controlled arterial hypertension in arrange appointments and were followed during one year. It was considered as a non-compliant those patients who did not attend to more than $20 \%$ of the arrange appointments. In case of not atten dance, we called the patient by telephone to make a new appointment.

Results: it was included 129 patients (the mean was 7.3 controls in a year). There were $11.2 \%$ non-com pliance to the clinics and $24.8 \%$ were non-compliant, although $51.2 \%$ did not attend to any appointments.

Men were more non-compliant than women (36.2 vs $18.3 \%$ ) and people below 50 years old (66.7\% non-compliant). It was not observe diffe rences in relation with cultural level, civil state or work situation, but there were differences related with cohabitation $(45.5 \%$ lived alone vs $20.2 \%$ and $22.2 \%$ if they lived in family or with another peo ple). Previous non-compliant patients were also more non-compliant (34.5 vs $16.9 \%$ ).

It was not found any differences in compliance depending on clinic, the appointment's time, inter val between the appointments or distance since the latest on-demand clinic.

Conclusions: it does not exist a clear type of non-compliant hypertensive patient to regular 
probablemente habría que estar alerta y/o tomar medidas anticipatorias en varones jóvenes y pacientes con incumplimientos previos

Palabras clave: Consulta concertada. Incumpli miento. Hipertensión arterial. controls, but it would probably have to be alert and take anticipatory actions in young men and patients with previous non compliance appointments.

Key words: Arrange appointments. Non-com pliance. Hypertension.

\section{INTRODUCCIÓN}

La hipertensión arterial (HTA) es una enfermedad crónica de alta prevalencia en nuestro medio ${ }^{1-4}$, estando demostrada su asociación con la morbimortalidad cardiovascular ${ }^{5,6}$, permitiendo su tratamiento y control la disminución de la incidencia de las mismas de un modo eficiente ${ }^{7,8}$. La HTA supone, así mismo, uno de los procesos que ocasionan mayor consumo de recursos en general ${ }^{9,10}$ y un alto número de consultas, principalmente en el ámbito de Atención Primaria ${ }^{11,12}$.

La consulta programada o concertada, entendiéndose como tal la que se produce tras un acuerdo entre el profesional y el paciente ${ }^{13}$, supone una de las herramientas básicas de la Atención Primaria ${ }^{14,15}$, adecuándose claramente sus objetivos al seguimiento y control de procesos crónicos como la HTA $^{4,16}$.

Por otra parte, el cumplimiento, observancia o adherencia se define como la medida en que el paciente asume las normas o consejos dados por el profesional en cuanto a los cambios en el estilo de vida, toma de medicación, asistencia a revisiones, etc. ${ }^{17,18} \mathrm{o}$ siendo quizás el cumplimiento a las citaciones uno de los aspectos menos estudiados a pesar de que al faltar a las citas, los pacientes se privan de los servicios, desorganizan la atención y la relación, quitan tiempo potencial a otros pacientes y aumentan los costes sanitarios ${ }^{19}$.

En este marco, el presente estudio tiene como objetivos principales estudiar el grado de cumplimiento a las citas de una consulta concertada de un grupo de pacientes hipertensos y las posibles variables sociodemográficas y organizativas implicadas en el mismo.

\section{MATERIAL Y MÉTODOS}

La población estudio correspondió a los pacientes diagnosticados de HTA de un cupo de Medicina General del Centro Salud "Sillería" (Toledo) y que estaban incluidos en la consulta concertada siguiendo los controles establecidos en el programa del centro. Quedaron excluidos del estudio aquellos pacientes que estando diagnosticados de HTA no estaban incluidos en dicha consulta, bien por motivo de su dificultad en acudir al centro (p. ej., incluidos en programa de atención domiciliaria), dificultades relacionadas con su trabajo, falta de interés en dicho tipo de atención o por cualquier otro motivo.

Consideramos consulta concertada a aquélla que es acordada por el profesional y el paciente en la visita previa y que queda recogida en el libro de citaciones del profesional y en la tarjeta de citaciones del paciente, quedando definidas y registradas la consulta (médica o de enfermería), el día y la hora de citación, siguiendo la metodología propuesta por algunos autores ${ }^{13,20}$.

La periodicidad de los controles y el profesional que realizaba cada visita concreta estaban definidos por el programa existente en el centro.

Los pacientes fueron seguidos durante 1 año, considerándose como incumplidores a aquéllos que faltaron al menos al $20 \%$ de las visitas establecidas siguiendo los límites mencionados por Sackett ${ }^{21}$, al ser estos los más aceptados en la literatura. En los casos de incumplimiento se contactó con el paciente por vía telefónica, interesándose por la causa del incumplimiento y facilitándosele una nueva citación. En los casos en los que no fue posible el contacto telefónico, se aprovechó la siguiente consulta a demanda del paciente para recoger el motivo de no asistencia a la cita y reiniciar el proceso de citaciones periódicas.

Las características demográficas fueron recogidas de la historia clínica de los pacientes y se relacionan con el carácter de cumplidor o no cumplidor del paciente. Las variables referentes a cada consulta concreta (hora citación, consulta, tiempo desde citación previa,...) fueron recogidas en cada una de las citaciones y se relacionan con la asistencia o no a esa citación concreta.

Los datos recogidos fueron introducidos en una base de datos del paquete estadístico R-Sigma para su estudio estadístico. 


\section{RESULTADOS}

Fueron incluidos en el estudio un total de 129 hipertensos seguidos en consulta concertada, siendo el $64,3 \%$ mujeres y teniendo una edad media de 68,4 años (D.S. 9,5 años). En total generaron 941 consultas con una media de 7,29 consultas/paciente/año.

El 24,8\% de los pacientes cumplieron el criterio de paciente incumplidor al finalizar el año de seguimiento del estudio, si bien hasta el $51,2 \%$ de los pacientes había faltado a alguna de las citaciones acordadas durante el periodo. Del total de consultas, existió incumplimiento en el $11,16 \%$ de las mismas.

Fueron incumplidores el 36,2\% de los varones frente al $18,3 \%$ de las mujeres. En la figura 1 aparece el porcentaje de incumplimiento según los grupos de edad, siendo los menores de 50 años los que presentan un mayor porcentaje de incumplidores $(66,7 \%)$.

No se observaron diferencias importantes en función del nivel de estudios (22,2\% incumplidores en analfabetos, $24,4 \%$ en estudios primarios y $28,0 \%$ en estudios medios/superiores), estado civil (30,0\% en solteros, $21,5 \%$ en casados y $30,0 \%$ en otros), ni situación laboral ( $30,0 \%$ en activos, $26,9 \%$ en pensionistas y $19,1 \%$ en amas de casa). En la figura 2 se expone el porcentaje de incumplidores según convivencia.

Los pacientes con incumplimientos previos fueron más incumplidores $(34,5 \%)$ que aquéllos que no tenían en su historia clínica antecedentes de incumplimiento $(16,9 \%)$.

No se evidenciaron diferencias importantes en el grado de incumplimiento a las citas en función de la consulta $(10,5 \%$ incumplimiento en consulta médica y $11,4 \%$ en consulta de enfermería) ni hora de citación ( $11,4 \%$ de incumplimiento a primera hora, $11,4 \%$ en horas intermedias y $8,3 \%$ al finalizar la mañana).

En la figura 3 se recogen los datos de incumplimiento según la época del año de dicha citación. La figura 4 refleja el porcentaje de incumplimiento en relación con el intervalo desde la última citación y desde la última consulta. Por último, las citas en las que existía antecedente de incumplimiento presentaron un mayor porcentaje de incumplimiento $(16,4 \%)$ que aquéllas en las que no existía dicho antecedente $(5,5 \%)$ (Fig. 5).

\section{DISCUSIÓN}

Diversos autores coinciden en que una de las principales causas del mal control de los pacientes hipertensos es la falta de cumplimiento ${ }^{22-25} \mathrm{y}$, si bien está demostrado que la asistencia a citas no es un

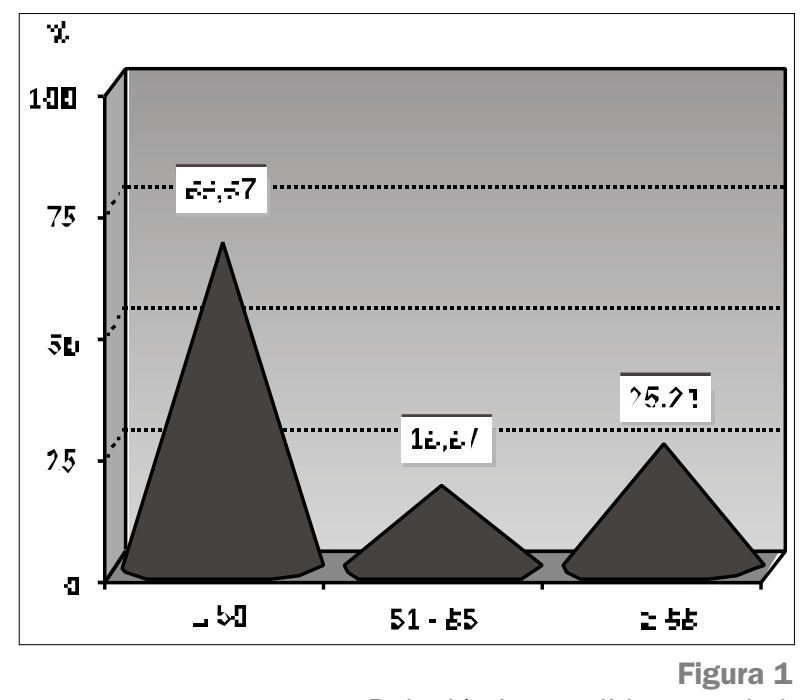

Relación incumplidores - edad.

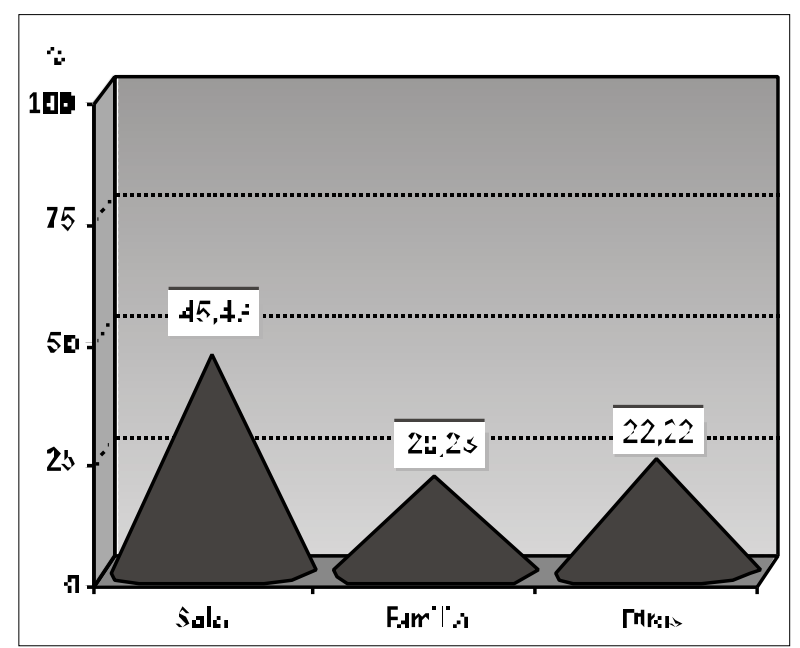

Figura 2

Relación incumplidores - convivencia.

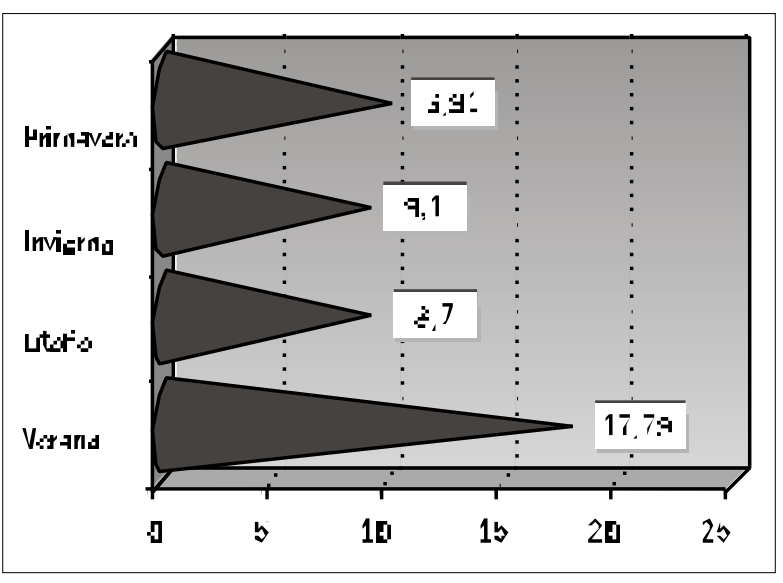

Figura 3

Relación cumplimiento - época del año. 


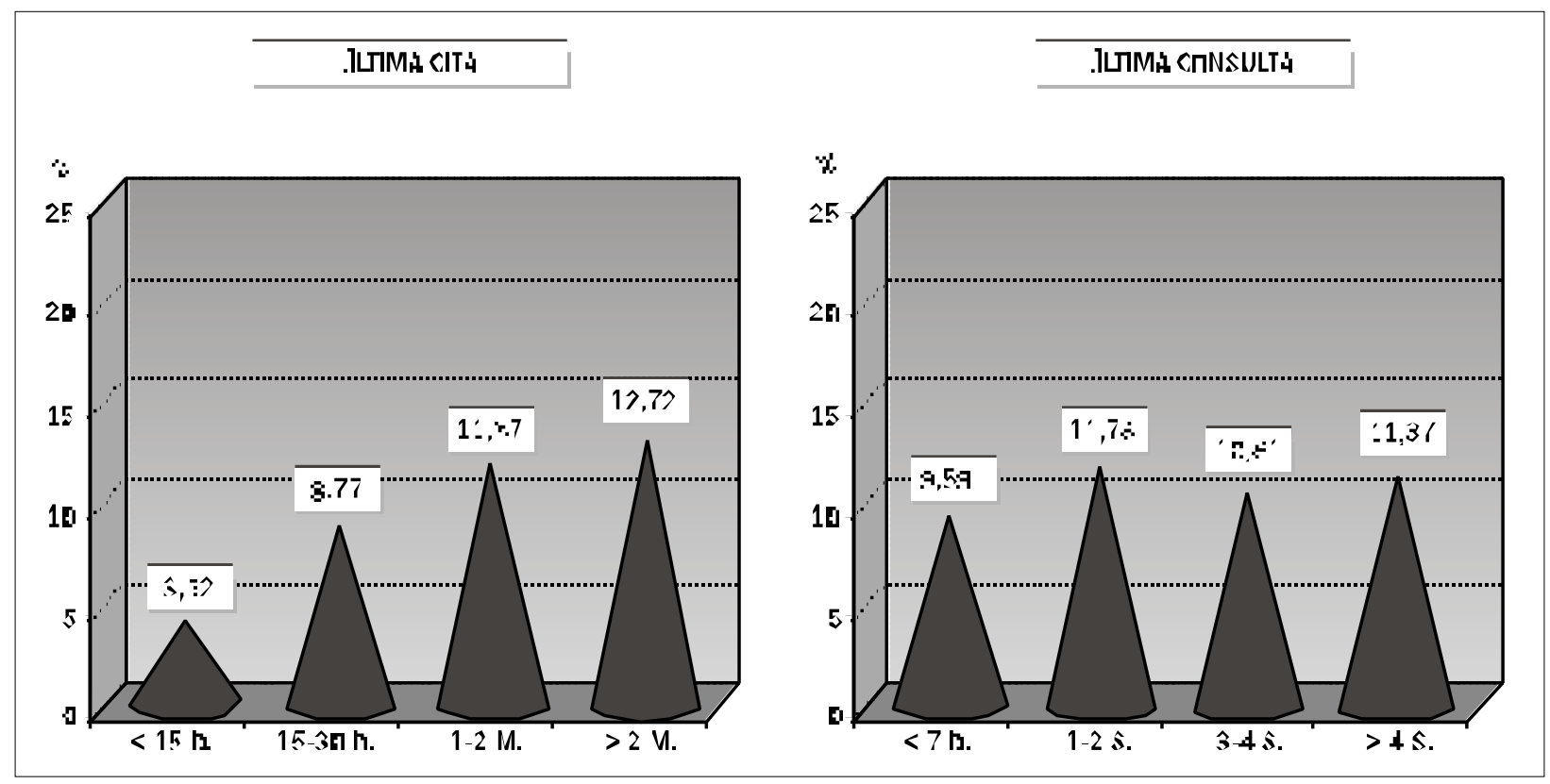

Figura 4

Relación cumplimiento con última cita y última consulta.

método válido para valorar el cumplimiento terapéutico en estos pacientes ${ }^{26-28}$, no es menos cierto que los pacientes que no acuden a las citas concertadas raramente realizan las indicaciones terapéuti$\operatorname{cas}^{21,29,30}$ y que existen investigaciones que relacionan el incumplimiento a citas con el grado de control de la $\mathrm{HTA}^{31-33}$. Además, la falta de asistencia a los controles concertados supone un obstáculo importante para la eficacia de las intervenciones sanitarias ${ }^{19}$.

El porcentaje de incumplidores y de incumplimiento a citas específicas encontrado en nuestro estudio se encuentra dentro del rango de los obtenidos por otros autores realizados en España dentro del ámbito de Atención Primaria ${ }^{26-28,31,32,34-42}$, si bien esta comparación debe realizarse con cautela debido a que entre los mismos existen diferencias en cuanto a definición de incumplimiento (algunos sólo consideran incumplimiento por encima del $50 \%$ de las citas), tiempo de seguimiento (la mayoría de los estudios son en 6 meses), características de las consultas (en algunos casos se trata de consultas específicas de HTA), etc. Debe tenerse en cuenta que la relación médico-paciente es un factor importante en el cumplimiento de las $\operatorname{citas}^{40,42}$, lo cual también limita la comparabilidad y que, además, el presente estudio está realizado con pacientes de una única consulta, lo cual puede representar una limitación en cuanto a su generalización.

Las razones del incumplimiento no son bien conocidas, dando lugar a diversas teorías ${ }^{43-45}$, por lo

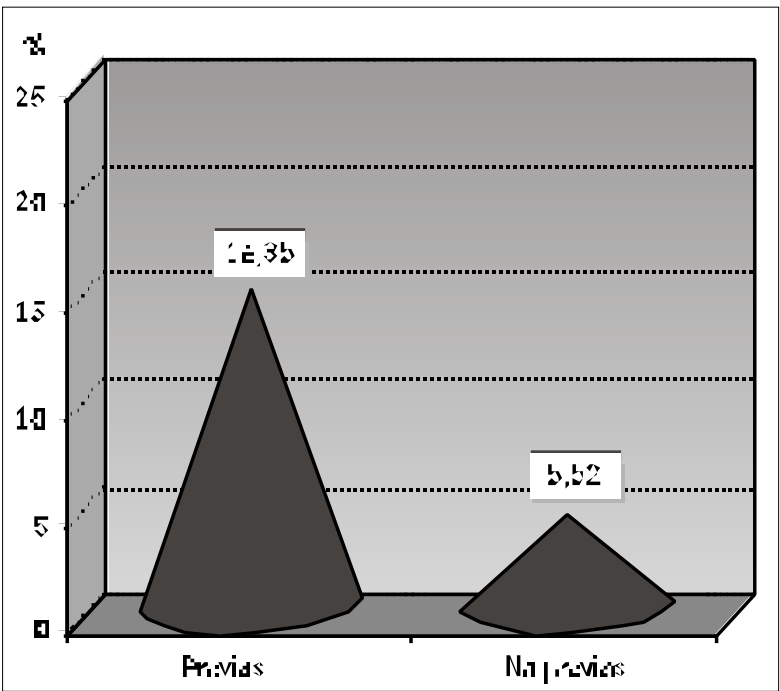

Figura 5

Relación incumplimiento - incumplimientos previos.

que es de interés evaluar estas ausencias a las citaciones y los factores que intervienen ${ }^{20,36,38,46}$. No existe un perfil definido del paciente incumplidor en general ${ }^{47,48}$, si bien si existe algún trabajo que, como nosotros, encuentra un mayor incumplimiento a las citas en varones jóvenes ${ }^{37,40,49,50}$, quizás debido a una mayor desmotivación hacia la enfermedad de este grupo de edad ${ }^{37,49}$. 
El mayor porcentaje de incumplidores encontrado en personas que viven solas podría estar ocasionado por la falta de apoyo/estímulo del entorno familiar $^{29,51,52}$.

El hecho de presentar un mayor porcentaje de incumplimientos en los que tenían incumplimientos previos ya fue descrito en otro estudio realizado en nuestro Centro de $\mathrm{Salud}^{38}$, quizás en relación con que entre las principales causas del incumplimiento alegadas por los pacientes estén el desinterés y el olvi$\mathrm{do}^{37,38}$, pudiendo sugerirse que aquellos pacientes que durante un largo periodo se han comportado como buenos cumplidores puedan ofrecer garantías razonables a largo plazo ${ }^{53}$, aunque algunos autores detectan que el incumplimiento a largo plazo tiende a ser mayor $^{29,54}$.

Destacar que, a diferencia de lo expresado por otros autores ${ }^{19,38,55}$, no hemos encontrado grandes diferencias de cumplimiento con relación al tiempo transcurrido desde la última citación o la última consulta. Tampoco hemos encontrado diferencias marcadas en el cumplimiento entre consulta médica y de enfermería, estando demostrado que en ésta se puede realizar una atención continuada y eficaz de la atención ${ }^{56,57}$.

Por último, a pesar del porcentaje de incumplimiento a citas concertadas, no debemos olvidar que la consulta concertada ha demostrado mayor efecti- vidad y aceptación por los pacientes que la consulta a demanda ${ }^{36,58-60}$ y que existen medidas que han evidenciado ser eficaces para aumentar el cumplimiento a las $\operatorname{citas}^{61-63}$. Unido a esto, parece de interés facilitar la asistencia a una nueva consulta tras un incumplimiento, lo cual podría realizarse a través de estrategias similares a la seguida por nosotros (contacto telefónico y facilitación de nueva citación).

Concluimos que no existe un perfil definido del paciente incumplidor a citas, pero que probablemente habría que estar alerta en los varones jóvenes y en las personas con incumplimiento previo para, quizás en ellas, tomar medidas anticipadas como el recordatorio telefónico para la prevención del incumplimiento.

\author{
CORRESPONDENCIA: \\ R. Orueta Sánchez \\ Centro de Salud "Sillería" \\ C/ Sillería $\mathrm{s} / \mathrm{n}$ \\ 45001 Toledo \\ Tfno.: 925215054
}

\section{Bibliografía}

1. Pardell H. La hipertensión arterial en España; Compendio de trabajos epidemiológicos sobre la hipertensión arterial. Liga Española para la Lucha contra la Hipertensión Arterial. ( $2^{\mathrm{a}}$ ed). Madrid, 1986.

2. Instituto de Estudios Médico-Sanitarios Hoechts. Estudio prospectivo Delphi sobre escenarios actuales y futuros del control de la hipertensión y sus complicaciones. Madrid, 1993.

3. Treserras R, Pardell H. Prevalencia e importancia sanitaria de la hipertensión arterial. Ann Med Intern 1970; 7: 1-6.

4. Ministerio de Sanidad y Consumo. Control de la hipertensión arterial en España, 1996. Ministerio de Sanidad y Consumo. Madrid, 1996.

5. Report of a WHO Expert Committe. Hypertension Control. WHO. Technical Report Series 862. Ginebra: World Health Organization, 1996.

6. Kannel WB. Blood pressure as a cardiovascular risk factor. Prevention and treatment. JAMA 1996; 275: 1571-6.

7. MacMahon SW, Cutter JA, Neaton JD, Payne GH. Relationship of blood pressure to coronary and stroke morbidity and mortality in clinical trials and epidemiological studies. J Hypertens 1986; 4: 14-7.
8. Brown AD, Garber AM. Cost effectiveness of coronary heart disease prevention strategies in adults. Pharmacoeconomics 1998; 14: 27-48.

9. Badia X, Rovira J, Trasseras R, Trinxet C, Segú J, Pardell H. El coste de la hipertensión en España. Med Clin (Barc) 1992; 99: 769-73.

10. Badia X, Segi JL. Farmacoeconomía y tratamiento de la hipertensión arterial. Cuadernos de Gestión 1998; 4: 181-90.

11. Lombardero E. Estudio de la demanda asistencial en una zona rural de Asturias. Aten Primaria 1985; 2: 73-9.

12. Díaz Caneja MA, Santamaría T, Polo I, Lahoz F, Seguido P, Rodríguez E. Estudio prospectivo de la consulta programada de enfermería en un Centro de Salud urbano. Aten Primaria 1991; 8: 460-4.

13. Arribas L. La atención médica a demanda y programada. En: De la Revilla L. La consulta del medico de familia: la organización de la práctica diaria. Madrid: Jarpio, 1992.

14. Jiménez J. Programación y protocolización de actividades. Monografías Clínicas en Atención Primaria. Barcelona: Doyma, 1990.

15. Bellan JA. La consulta del medico de familia; cómo organizar el trabajo (2). Consulta programada y otras activida- 
des. En: Gallo FJ. Manual del Residente de Medicina Familiar y Comunitaria. semFYC. Madrid, 1997.

16. Abellan J, Martínez A. La Hipertensión Arterial en Atención Primaria: recomendaciones para su detección, evaluación y control. Liga Española para la Lucha Contra la Hipertensión Arterial. Madrid, 1991.

17. Haynes RB. Introduction. En: Haynes RB, Taylor DW, Sackett DL, eds. Compliance in health care. Baltimore: The Johns Hopkins University Press, 1979; 1-7.

18. Merino J, Gil VF, Belda J. Métodos de conocimiento y medidas de la observancia. La observancia en el tratamiento de la hipertensión arterial. Barcelona: Doyma, 1993.

19. Macharia WN, León G, Rowe BH, Stephenson B, Haynes B. An overview of interventions to improve compliance with appointment keeping for medical services. JAMA 1992; 267: 1813-7.

20. Ruiz-Giménez JL, Uriarte A. Actividad clínica médica. En: Organización del Equipo de Atención Primaria. Ministerio de Sanidad y Consumo. Madrid, 1990.

21. Sackett D, Haynes RB, Tugwell P. Cumplimiento. En: Epidemiología clínica: una ciencia básica para la medicina clínica. Madrid: Díaz de Santos, 1989; 259-89.

22. The Sixth Report of the Joint National Committe on Prevention, Detection, Evaluation, and Treatment of High Blood Pressure. National Institutes of Heart. National Lung and Blood Institute. 1997.

23. Haynes RB, Mattson ME, Chobaniman AV. Managament of patient compliance in the treatment of Hypertension. Report of the NHLBI Working Group. Hypertension. 1982; 4: 415-23.

24. Haynes RB, Sackett DL, Gibson DL, Taylor DW, Hackett $\mathrm{B}$, Roberts $\mathrm{R}$ et al. Improvement of medication compliance in uncontrolled hypertension. Lancet 1976; 1: 1265-8.

25. Abellan J, Leal M, García-Galbis JA. Papel de la Atención Primaria en el control de la presión arterial. Hipertensión. 1999; 16: 147-54

26. Gil V, Pineda M, Martínez JL, Belda J, Santos MC, Merino J. Validez de 6 métodos indirectos para valorar el cumplimiento terapéutico en hipertensión arterial. Med Clin (Barc) 1994; 102: 532-6.

27. Piñero F, Gil V, Donis M, Orozco D, Pastor R, Merino J. Validez de 6 métodos indirectos para valorar el cumplimiento terapéutico en hipertensión arterial. Aten Primaria 1997; 19: 372-5.

28. Márquez E, Gutiérrez C, Jiménez C, Franco C, Baquero C, Ruiz R. Observancia terapéutica en la hipertensión arterial. Validación de métodos indirectos que valoran el cumplimiento terapéutico. Aten Primaria 1995; 16: 496500 .

29. Sackett D, Snow JC. The magnitude of compliance and non compliance. En: Autores Compliance in Health Care. Baltimore: Johns Hopkins University Press 1979; 11-22.

30. Grupo de Trabajo en hipertensión. Observancia terapéutica en el paciente hipertenso. Sociedad Española de Hipertension. Liga Española para la Lucha contra la Hipertensión Arterial. Madrid, 1996.

31. Dalfo A, Botey A, Buil P, Esteban J, Gual J, Revert L. Estudio del seguimiento y control del paciente hipertenso en la asistencia primaria y hospitalaria. Aten Primaria 1987; 4: 233-9.

32. Fornells JM, Balaguer I. Grupo de control de la hipertensión del programa Cronicat. Control de la hipertensión en el medio rural: 18 meses de seguimiento (programa Cronicat). Med Clin (Barc) 1987; 89: 450-5.

33. Márquez E, Casado JJ, Ramos J, Saenz S, Moreno JP, Celotti B, et al. Influencia del cumplimiento terapéutico en los niveles de presión arterial en el tratamiento de la hipertensión arterial. Hipertensión 1998; 15: 133-9.

34. Gil V, Pomares R, Pomares A, Alberola T, Belda J, Merino J. Indicadores de actividad en el seguimiento de una unidad de hipertensión arterial durante 5 años. Aten Primaria 1993; 12: 264-8.

35. Gil V, Belda J, Muñoz C, Martínez JL, Soriano E, Merino J. Validez de cuatro métodos indirectos que valoran el cumplimiento terapéutico en la hipertensión arterial. Rev Clin Esp 1993; 193: 363-7.

36. Iturrioz P, Mariñelarena E, Guillen P, Abad I, Soricano M, Arrieta M, et al. Evaluación del cumplimiento de la citación y del control de la tensión arterial en un grupo de hipertensos. Aten Primaria 1991; 8: 212-6.

37. Gil V, Martínez JL, Muñoz C, Alberola T, Belda J, Merino J. Estudio durante cuatro años de la observancia terapéutica de pacientes hipertensos. Rev Clin Esp 1993; 193: 351-6.

38. Sánchez C, Gómez-Calcerrada R, Gonzalez M, Orueta R. Causas de incumplimiento y factores asociados en una consulta concertada. Aten Primaria 1996; 17: 34-8.

39. Pastor RM. Estudio del cumplimiento a citas concertadas de pacientes con patologías crónicas: hipertensión arterial, diabetes y dislipemia. Tesis Doctoral. Departamento de Medicina. Universidad Alicante, 1997.

40. Iruela $\mathrm{T}$, Juncosa $\mathrm{S}$. Factores que influyen en el seguimiento de los pacientes hipertensos. Aten Primaria 1996; 17: 332-7.

41. Delgado E, Hernández E, Izquierdo JM, Landa V, Lejarza R, Lopez V et al. Evaluación del grado de cumplimiento del protocolo de hipertensión arterial. Aten Primaria 1990; 7: 635-40.

42. Piñero F, Gil V, Pastor R, Merino J. El incumplimiento de las citas programadas en los pacientes hipertensos: perfil del incumplidor. Rev Clin Esp 1998; 198: 669-72.

43. Leventhal H, Cameron L. Behavioral theories and the problem of compliance. Patient Educ Couns 1987; 10: 117-38.

44. Eraker SA, Kirscht JP, Becker NH. Understanding and improving patient compliance. Ann In Med 1984; 100: 258-68.

45. Reichgott MJ, Simons-Morton BG. Strategies to improve patient compliance with anthypertensive therapy. Primary Care 1983; 10: 21-7.

46. Calvo E, Frieyro JE, Álvarez S, et al. Estudio prospectivo del cumplimiento de la cita en un programa doente de Medicina Familiar y Comunitaria. VIII Jornadas Nacionales de Medicina Familiar y Comunitaria. Valladolid, 1988.

47. Blackwell B. Patient compliance. N Engl J Med 1973; 289: 249-52.

48. Abellan J. La observancia del tratamiento. Hipertension 1994; 11: 1-2.

49. Weingarten MA, Cannon BS. Age as a major factor affecting adherence to medication for hypertension in a General Practice population. Family Practice 1988; 5: 294-6.

50. Degoulet P, Menard J, Vu HA, Goldmard JL, Devries C, Chatellier $\mathrm{G}$ et al. Factors predictive of attendance at clinic and blood pressure control in hypertension patients. Brit Med J 1983; 287: 88-93.

51. Urquizar J, Pinteño P, Ballesteros P, Antequera JA, Mellado B, García MC. Factores sociofamiliares que influyen en el cumplimiento farmacológico del paciente hipertenso. XVI Congreso Sociedad Española de Medicina Familiar y Comunitaria. Granada, 1996.

52. Green DW, Levine DM, Deeds S. Clinical trials of health education for hypertensive outpatients. Prev Med 1975; 4: 417-25.

53. Martínez Amenos A. La observancia en el tratamiento de la hipertensión arterial. Barcelona: Doyma, 1993.

54. Gil V. La hipertensión arterial en Atención Primaria. Tesis doctoral. Departamento de Medicina. Universidad de Alicante, 1991

55. Lichtenstein MJ, Sweetnam PM, Elwood PC. Visit frecuency for controlled essential hypertension: General Practicer opinions. J Fam Pract 1986; 23: 331-6.

56. Gleichmann SI, Gleichmann VM, Mannebach HJ, et al. Educating nurses in blood pressure measurement and hypertension control. J Hypertens 1989; 7: S99-S102. 
57. González L. Adhesión terapéutica y conocimiento en hipertensión de pacientes incluidos en consulta de enfermería. Aten Primaria 1993; 12: 469-73.

58. Kotchen JM, Mckean HE, Jackson S, Thayer S. Impact of rural high pressure control program on hypertension control and cardiovascular disease mortality. JAMA 1986; 255: 2177-82.

59. Meinchenbaun D, Turck DC. Factors affecting adherence. En: Meinchenbaun D, Turck DC eds. Facilitating treatment adherence. A Practitioner's guidebook. New York: Plenum Press, 1987; 41-7.
60. Rastam L, Berglund G, Isacsson SO, Ryden L. The Skaraborg Hypertension Projet II. Feasibility of a medical care program for hypertension. Acta Med Scand 1986; 219: 249-60.

61. McDowell I, Newell C, Rosser W. A randomized trial of computerized reminders for blood pressure screening in Primary Health Care. Med Care 1989; 27: 297-305.

62. Nessman DG, Carnahan JE, Nugent CA. Increasing compliance. Patient-operated hypertension group. Arch Intern Med 1980; 140: 1427-30.

63. Levine DM, Green DW, Deed S. Health education for hypertensive patients. JAMA 1979; 241: 1700-3. 